\title{
Structural and electronic interaction at CuO-hexa-peri-hexabenzocoronene hybrid interface
}

\author{
Bharti Singh, ${ }^{1}$ B. R. Mehta, ${ }^{1, a)}$ M. Singh, ${ }^{1}$ Govind Gupta, ${ }^{2}$ L. Dössel, ${ }^{3}$ X. Feng, ${ }^{3}$ and \\ K. Müllen ${ }^{3}$ \\ ${ }^{1}$ Thin Film Laboratory, Department of Physics, Indian Institute of Technology Delhi, \\ New Delhi 110016, India \\ ${ }^{2}$ Surface Physics Group, National Physical Laboratory (CSIR), New Delhi 110012, India \\ ${ }^{3}$ Max-Planck Institute for Polymer Research, D-55128 Mainz, Germany
}

(Received 16 November 2010; accepted 4 January 2011; published online 14 February 2011)

\begin{abstract}
Interfacial interaction at hybrid interfaces results in structural and electronic properties different from organic and inorganic components. In this study, $\mathrm{x}$-ray photoelectron spectroscopy analysis carried out on $\mathrm{CuO}$-hexa-peri-hexabenzocoronene (HBC) bilayers shows the appearance of an additional $\mathrm{C} 1 \mathrm{~s}$ peak at lower energy due to $\mathrm{Cu}-\mathrm{C}$ interaction. Spectroscopic ellipsometry investigation shows that interfacial layer $(\sim 4 \mathrm{~nm})$ has absorption features related to $\mathrm{Cu}-\mathrm{C}$ bonding, modified HBC valence states, and $\mathrm{Cu}^{2+}-\pi$ electron interaction. The observed resistive switching property of the $\mathrm{CuO}-\mathrm{HBC}$ layers is considerably different from junction properties of $\mathrm{HBC}$ and $\mathrm{CuO}$ layers and is directly related to the hybrid interfacial layer. () 2011 American Institute of Physics. [doi:10.1063/1.3544937]
\end{abstract}

Understanding the interfacial properties of an organicinorganic (OI) hybrid junction is scientifically challenging due to large dissimilarities in the structural and electronics nature of the two materials. ${ }^{1}$ Recent reports suggest that hybrid interfaces will open up possibilities for devices such as organic light emitting diodes, thin film field-effect transistors, and photovoltaic devices. ${ }^{2-5}$ By controlling the properties of organic and inorganic layers, the interfacial properties of the hybrid interfaces and the device characteristics can be fine tuned and controlled.

This letter reports the study of the interface between inorganic $\mathrm{CuO}$ semiconductor and organic hexa-perihexabenzocoronene (HBC) layers and the effect of hybrid interfacial layer on the optical and resistive switching properties. HBC is a well known planar polycyclic aromatic hydrocarbon molecule and can be regarded as disk-shaped hydrogen terminated graphene fragment, having delocalized electrons on both sides of the molecular plane. HBC molecules segregate themselves into columnar superstructures due to $\pi-\pi$ interactions of the aromatic cores. It is known to exhibit high intrinsic charge carrier mobilities of up to $1 \mathrm{~cm}^{2} / \mathrm{V} \mathrm{s}$ in its bulk form with a highest occupied molecular orbital and lowest unoccupied molecular orbital (LUMO) gap of $2.8 \mathrm{eV}^{6}{ }^{6} \mathrm{HBC}$ layer has been used as promising interfacial layer for increasing open circuit voltage of GaAs based Schottky barrier solar cells. ${ }^{7}$ On the other hand, $\mathrm{CuO}$ is a p-type inorganic semiconductor having monoclinic structure with an optical band gap of $1.2 \mathrm{eV}^{8}$ The hole mobility of $\mathrm{CuO}$ is $0.1 \mathrm{~cm}^{2} / \mathrm{V} \mathrm{s}$. $\mathrm{CuO}$ has excellent photovoltaic, electrochemical, and catalytic properties. ${ }^{9}$ The central objective of this study is to investigate the structural and electronic changes at the $\mathrm{CuO}-\mathrm{HBC}$ heterointerface for understanding the switching mechanism in a bilayer resistive memory device.

\footnotetext{
a) Author to whom correspondence should be addressed. Electronic mail: brmehta@physics.iitd.ac.in.
}

In this study, deposition of thin organic layer of $\mathrm{HBC}$ $(\sim 20 \mathrm{~nm})$ onto the copper base has been carried out using thermal evaporation technique. The synthesis of HBC is described elsewhere. ${ }^{10} \mathrm{~A}$ thin film of $\mathrm{CuO}(\sim 400 \mathrm{~nm})$ was deposited over $\mathrm{HBC}$ layer using rf-magnetron sputtering from a $\mathrm{Cu}_{2} \mathrm{O}$ target $(99.99 \%$ purity). The sputtering was carried out at room temperature with an applied rf power of $100 \mathrm{~W}$. Thereafter, a titanium top electrode $(\sim 100 \mathrm{~nm})$ was deposited using thermal evaporation technique through a shadow mask onto the copper oxide layer. Junction properties of Ti-CuO-HBC $(20 \mathrm{~nm})-\mathrm{Cu}$ have been studied using 6517-A electrometer from Keithley, Ohio.

X-ray photoelectron spectroscopy (XPS) was carried out using an ultra high vacuum XPS system (Model 1257) from Perkin Elmer, Minnesota. The $\mathrm{Cu}-\mathrm{HBC}$ and $\mathrm{CuO}-\mathrm{HBC}$ sample for XPS studies were prepared by depositing a thin layer of $(\sim 10 \mathrm{~nm}) \mathrm{Cu}$ and $\mathrm{CuO}$ onto a $\mathrm{HBC}$ layer deposited on glass substrate. The optical properties of $\mathrm{CuO}(10 \mathrm{~nm})$ HBC sample were studied using spectroscopic ellipsometry (SE) in the wavelength range of $200-1000 \mathrm{~nm}$ using an M-2000F ellipsometer from J. A. Woollam Co., Inc., Nebraska.

Figure 1(a) shows the $\mathrm{C} 1 s$ spectra of $\mathrm{CuO}-\mathrm{HBC}, \mathrm{HBC}$, and $\mathrm{Cu}-\mathrm{HBC}$ sample. It was observed that in addition to the $\mathrm{C} 1 s$ peak at $284.6 \mathrm{eV}$ observed in all samples, an additional peak at $282.7 \mathrm{eV}$ is observed in the case of the $\mathrm{CuO}-\mathrm{HBC}$ sample. The low intensity peak at lower energy in comparison with the main $\mathrm{C} 1 s$ peak indicates interaction at the $\mathrm{CuO}-\mathrm{HBC}$ hybrid interface. Electronegativity difference between $\mathrm{Cu}(1.9)$ and $\mathrm{C}(2.5)$ results in charge transfer from $\mathrm{Cu}$ to $\mathrm{C}$, causing an additional $\mathrm{C} 1 s$ peak at lower binding energy corresponding to a $\mathrm{Cu}-\mathrm{C}$ bond. In a similar study, fluorine groups have been shown to influence the electronic properties of $\mathrm{HBC}$ and the higher electronegativity of $\mathrm{F}$ (4.1) with respect to $\mathrm{C}$ resulted in electron transfer from $\mathrm{C}$ to $\mathrm{F}$, which shifts the $\mathrm{C} 1 s$ peak toward higher binding energy by about $2 \mathrm{eV} .^{11} \mathrm{~A}$ similar shift in binding energy has been explained due to the interface polarization of the valence 

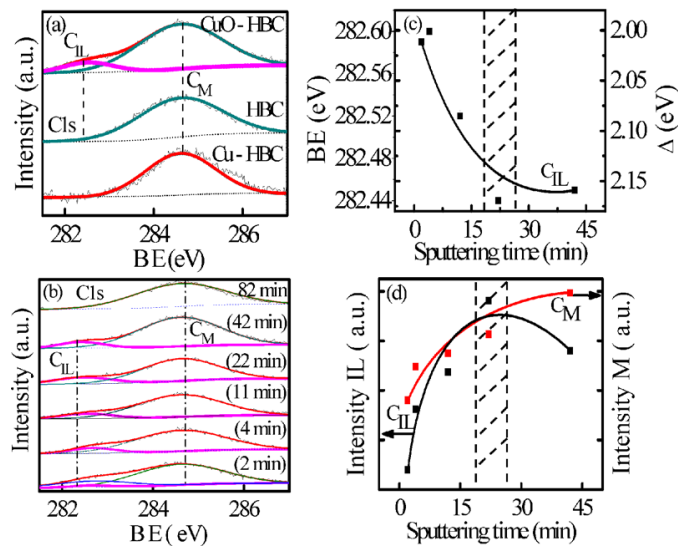

FIG. 1. (Color online) (a) $\mathrm{C} 1 \mathrm{~s}$ peak in $\mathrm{CuO}-\mathrm{HBC}, \mathrm{HBC}$, and $\mathrm{Cu}-\mathrm{HBC}$ sample. (b) Variation of $\mathrm{C} 1 s$ peak position in $\mathrm{CuO}-\mathrm{HBC}$ sample with depth profiling showing two $\mathrm{C} 1 s$ peaks [main peak $\left(\mathrm{C}_{\mathrm{M}}\right)$ at $284.60 \mathrm{eV}$ corresponding to $\mathrm{HBC}$ and interfacial layer $\left(\mathrm{C}_{\mathrm{IL}}\right)$ peak]. (c) Variation of binding energy (BE) and deviation $(\Delta)$ from the $\mathrm{C}_{\mathrm{M}}$ peak for $\mathrm{C}_{\mathrm{IL}}$ peak in CuO-HBC sample with depth profiling. (d) Intensity variation of $\mathrm{C}_{\mathrm{M}}$ and $\mathrm{C}_{\mathrm{IL}}$ peak with depth profiling.

density of states in Be/W system. ${ }^{12}$ To study the changes in C $1 s$ spectra across the hybrid interface, XPS depth profiling was carried out for CuO-HBC sample and the results are presented in Fig. 1(b). It was observed that the binding energy of the interfacial peak $\left(\mathrm{C}_{\mathrm{IL}}\right)$ decreases and the difference $(\Delta)$ between $\mathrm{C}_{\mathrm{IL}}$ and the main peak $\left(\mathrm{C}_{\mathrm{M}}\right)$ increases as one moves toward the interface up to about 20 min of sputtering [Fig. 1(c)]. The intensity of the $\mathrm{C}_{\mathrm{IL}}$ peak is observed to increase up to $20 \mathrm{~min}$ of sputtering, whereas the intensity of the $\mathrm{C}_{\mathrm{M}}$ peak at $284.6 \mathrm{eV}$ follows the opposite trend and continues to increase [Fig. 1(d)]. These results show that sample depth at $15-25 \mathrm{~min}$ of sputtering corresponds to the interfacial region [shown by the shaded portion in Figs. 1(c) and $1(\mathrm{~d})]$.

Spectroscopic ellipsometry was carried out onto $\mathrm{CuO}-$ HBC sample. The best fit to the experimental data in terms of ellipsometric angles $(\Psi)$ was obtained by incorporating an interfacial region of $(\sim 4 \mathrm{~nm})$ in the $\mathrm{CuO}-\mathrm{HBC}$ structure. Thickness and optical constants of the $\mathrm{CuO}$ top layer (TL), interfacial layer (IL), HBC bottom layer (BL) was obtained by fitting of the experimental data at three different incident angles using a four layer (CuO-IL-HBC-glass substrate) optical model. The absorption coefficient $(\alpha)$ versus energy $(\mathrm{h} \nu)$ plots for the top, bottom, and interfacial layers are shown in Fig. 2(b)-2(d), respectively. It was observed that the absorption spectra corresponding to $\mathrm{HBC}$ BL exhibits two peaks (marked as $3^{\prime}$ and $4^{\prime}$ ) and the IL absorption spectra show five peaks marked as (1, 2, 3, 4, and 5) [Figs. 2(c) and 2(d)]. This implies that the interface region has a number of distinct features in terms of absorption spectra. Electronic interaction between $\mathrm{CuO}$ and $\mathrm{HBC}$ in the interfacial region results in the appearance of additional peaks in the absorption spectra. The peak position in the absorption spectra for the HBC BL ( $3^{\prime}$ and $\left.4^{\prime}\right)$ and IL (1, 2, 3, 4 and 5) obtained is summarized in Table I. Peaks $3^{\prime}$ and $4^{\prime}$ observed at 2.75 and $3.5 \mathrm{eV}$ in the absorption spectra of $\mathrm{HBC} \mathrm{BL}$ are related to the I and II valence levels of HBC. A careful analysis of the peak position in the absorption spectra of the interfacial layer points toward its hybrid nature. Peaks 1 and 2 at 2.03 and 2.5 $\mathrm{eV}$ seems to be related to the band-to-band transition of $\mathrm{Cu}_{2} \mathrm{C}_{2}$ or $\mathrm{Cu}_{2} \mathrm{C}_{4}$ normally observed between 1.5 to $2.0 \mathrm{eV}^{13}$
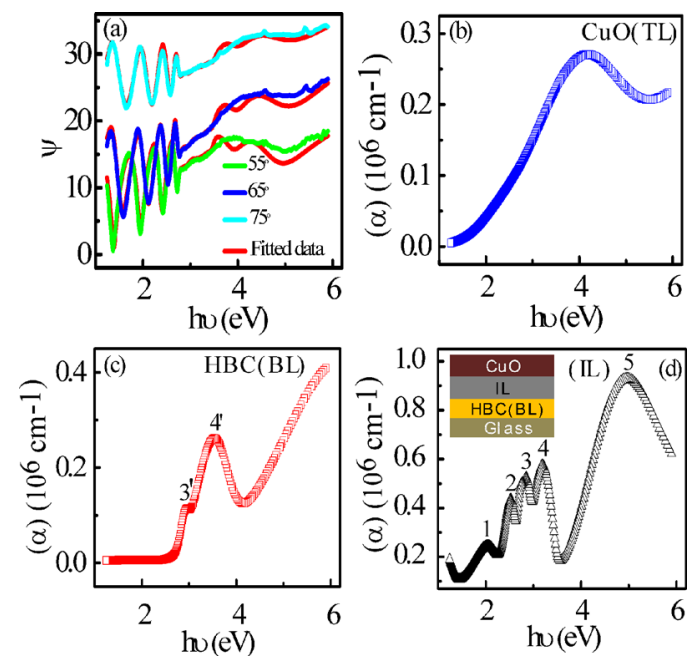

FIG. 2. (Color online) (a) Experimental and modeled ellipsometric angles " $\Psi$ " for CuO-HBC sample at three different incident angles of $55^{\circ}, 65^{\circ}$, and $75^{\circ}$ using four layer optical model [see inset of (d)]. Absorption spectra $(\alpha)$ for (b) $\mathrm{CuO} \mathrm{TL}$, (c) HBC BL, and (d) IL.

Peak positions marked as 3 and 4 correspond to modified I and II valence levels of HBC. Peak positions marked as 5 seem to be related to interaction of cation $\left(\mathrm{Cu}^{2+}\right)$ with the $\pi$ electron of aromatic chains of HBC. A similar absorption feature due to cation- $\pi$ electron has been presented in $\mathrm{Cu}^{2+}$-tryptophan system. ${ }^{14}$ Thus, the optical absorption spectra of the interfacial layer show structural and electronic interaction between the organic and inorganic components.

As the resistive switching phenomenon is known to be extremely sensitive to interfacial properties, ${ }^{15}$ the effect of interfacial interaction on $\mathrm{CuO}-\mathrm{HBC}$ hybrid interface has been studied by investigating resistive switching properties. For observing resistive switching phenomenon, an electroforming voltage is applied to metal-insulator-metal (MIM) structure, which results in a large change in resistance. Subsequently, the MIM structure can be switched from low resistance state (LRS) to high resistance state (HRS) upon application of reset point voltage $\left(\mathrm{V}_{\mathrm{R}}\right)$ and back to LRS on application of set point voltage $\left(\mathrm{V}_{\mathrm{S}}\right){ }^{16}$ If the LRS and HRS occur at the same voltage polarity, switching is referred to as unipolar; otherwise, bipolar.

Figure 3(a) shows the electroforming process performed on the pristine $\mathrm{Ti}-\mathrm{CuO}-\mathrm{HBC}-\mathrm{Cu}$ structure with a current compliance limit of $2 \mathrm{~mA}$ on applying a voltage of $0.44 \mathrm{~V}$. After electroforming, the Ti-CuO-HBC-Cu cell remains in the LRS and exhibits linear I-V characteristics up to a volt-

TABLE I. Comparison of peak position in absorption spectra for the HBC $\mathrm{BL}$ and IL with ultraviolet photoelectron spectroscopy results for the HBC sample (see Ref. 6). $\mathrm{E}_{\alpha} \mathrm{HBC}$ (BL) and $\mathrm{E}_{\alpha}$ (IL) correspond to the peak position in the absorption spectra for $\mathrm{BL}$ and IL, respectively, whereas $\mathrm{E}_{\mathrm{FL}}$ and $\mathrm{E}_{\mathrm{LUMO}}$ correspond to position of valence states with respect to the Fermi level and the lowest unoccupied molecular orbital.

\begin{tabular}{ccccc}
\hline \hline $\begin{array}{c}\mathrm{E}_{\alpha} \mathrm{HBC}(\mathrm{BL}) \\
(\mathrm{eV})\end{array}$ & $\begin{array}{c}\mathrm{E}_{\alpha}(\mathrm{IL}) \\
(\mathrm{eV})\end{array}$ & $\begin{array}{c}\mathrm{E}_{\mathrm{FL}} \\
(\mathrm{eV})\end{array}$ & $\begin{array}{c}\mathrm{E}_{\mathrm{LUMO}} \\
(\mathrm{eV})\end{array}$ & Peak assignment \\
\hline$\ldots$ & 2.03 & $\cdots$ & $\cdots$ & $\mathrm{Cu}_{2} \mathrm{C}_{2}$ \\
$\ldots$ & 2.51 & $\cdots$ & $\cdots$ & $\mathrm{Cu}_{2} \mathrm{C}_{4}$ \\
2.75 & 2.85 & 1.35 & 2.80 & First valence State $(\mathrm{HBC})$ \\
3.50 & 3.20 & 1.75 & 3.20 & $\begin{array}{c}\text { Second valence State }(\mathrm{HBC}) \\
\mathrm{Cu}^{2+}-\pi \text { interaction }\end{array}$ \\
$\cdots$ & 4.95 & $\cdots$ & $\cdots$ & \\
\hline \hline
\end{tabular}



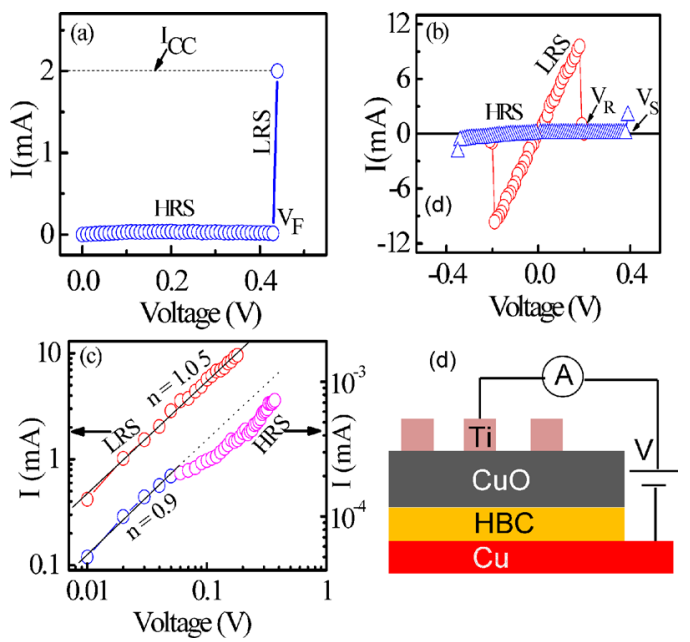

FIG. 3. (Color online) (a) Current-voltage characteristics for Ti-CuOHBC-Cu sample during the initial electroforming step, showing transition to LRS at forming voltage $\left(\mathrm{V}_{\mathrm{F}}\right)$ of $0.44 \mathrm{~V}$ with a current compliance limit $\left(\mathrm{I}_{\mathrm{CC}}\right)$ of $2 \mathrm{~mA}$. (b) I-V curve showing reversible and unipolar resistive switching for Ti-CuO-HBC-Cu sample. The sample switches from LRS to HRS at a reset voltage $\left(\mathrm{V}_{\mathrm{R}}\right)$ of $0.29 \mathrm{~V}$ and switches back to LRS at a set voltage $\left(\mathrm{V}_{\mathrm{S}}\right)$ of $0.39 \mathrm{~V}$. (c) Double logarithmic I-V plots showing the linear Ohmic behavior with slope of about 1.05 in the LRS. Linearity is observed in HRS at lower voltages with slope of about 0.9 ; deviation from this linearity is observed in HRS at higher voltages. (d) Schematic diagram of the Ti-CuO$\mathrm{HBC}-\mathrm{Cu}$ structure used for studying resistive switching properties.

age of $0.19 \mathrm{~V}$ as shown in Fig. 3(b). Upon increasing the voltage further, the sample switches to HRS at a reset voltage $\left(\mathrm{V}_{\mathrm{R}}\right)$ of $0.2 \mathrm{~V}$. Upon further increase in voltage bias, the sample switches back to LRS state upon application of a set point voltage $\left(\mathrm{V}_{\mathrm{S}}\right)$ of $0.39 \mathrm{~V}$. The typical resistance values in LRS and HRS are observed to be $(\sim 18 \Omega)$ and $(\sim 38.5$ $\times 10^{4} \Omega$ ) at a voltage of $0.1 \mathrm{~V}$. A high value of resistance ratio $\sim 2 \times 10^{4}$ between the HRS and LRS state is quite attractive from an application point of view. The resistive switching behavior was observed to be unipolar with reproducible I-V characteristics. Double logarithmic plots of the I-V curve in both LRS and HRS states are presented in Fig. 3(c). A I-V characteristic in LRS displays the Ohmic behavior with an approximate slope of 1.08. The conduction mechanism of the HRS at low voltage also follows Ohm's law, but appreciable nonlinearity is observed at high voltages. It does not seem to follow any single law and may be a combination of different possible current transport mechanisms such as trapping (detrapping) of space charge limited current and the formation (deformation) of multifilaments, as reported earlier. ${ }^{17}$ It may be noted that Ti-HBC-Cu and Ti$\mathrm{CuO}-\mathrm{Cu}$ structures showed linear and rectifying I-V characteristics, respectively (not shown here), and resistive switching behavior was observed to be completely absent. This confirms that hybrid interfacial layer is responsible for resistive switching phenomenon.

Furthermore, resistive switching characteristics of the Ti-HBC-CuO-Cu junction (not shown here) in which the $\mathrm{HBC}$ layer was vacuum evaporated onto a $\mathrm{CuO}$ layer shows higher electroforming voltage $(>50 \mathrm{~V})$ in comparison with $(0.44 \mathrm{~V})$ when the $\mathrm{CuO}$ layer is sputter deposited onto the HBC layer. XPS and SE results showed minimal modification at the interface in the former case (not shown here). Kinetic energy of copper or oxygen adatoms during sputter deposition of $\mathrm{CuO}$ onto a $\mathrm{HBC}$ layer seems to be an important factor controlling the extent of interfacial interaction. The degree of interfacial interaction at $\mathrm{CuO}-\mathrm{HBC}$ hybrid junction can be controlled by changing the energy of adatoms (sputtering voltage or deposition pressure) or by postdeposition heat treatment (time and duration). Such a study will help in further understanding the nature of the interfacial layer and the resistive switching mechanism. It may be mentioned that there are a number of reports on the resistive switching behavior in organic and inorganic layers. The resistive switching properties observed in the present study are due to hybrid interfacial layer and not due to $\mathrm{CuO}$ and $\mathrm{HBC}$ layer.

In conclusion, XPS analysis carried out on $\mathrm{CuO}-\mathrm{HBC}$ bilayers shows the appearance of an additional $\mathrm{C} 1 s$ peak at lower energy due to electron transfer from $\mathrm{Cu}$ to $\mathrm{C}$. Optical modeling based on SE data shows the formation of interfacial layer of about $4 \mathrm{~nm}$ thickness, with absorption features related to $\mathrm{Cu}-\mathrm{C}$ bonding, modified $\mathrm{HBC}$ valence states, and $\mathrm{Cu}^{2+}-\pi$ electron interaction. Electrical properties of MIM structures comprising of $\mathrm{CuO}-\mathrm{HBC}$ layers show that the interfacial layer is directly responsible for the observed resistive switching properties as junctions formed from the inorganic $\mathrm{CuO}$ layer and the organic $\mathrm{HBC}$ layer shows rectifying and Ohmic characteristics, respectively. This study presents an example in which the formation of hybrid interfacial layer results in electrical properties not observed in the organic and inorganic components.

One of the authors (Bharti Singh) is thankful to the Council of Scientific and Industrial Research, India for providing junior research fellowship. The authors would like to thank Mr. Amit Chauhan for carrying out X-ray photoelectron spectroscopy measurements.

${ }^{1}$ C. N. R. Rao, A. K. Cheetham, and A. Thirumurugan, J. Phys.: Condens. Matter 20, 083202 (2008).

${ }^{2}$ J. Lee, B.-J. Jung, J.-I. Lee, H. Y. Chu, L.-M. Do, and H.-Ku. Shim, J. Mater. Chem. 12, 3494 (2002).

${ }^{3}$ G. B. Murdoch, M. Greiner, M. G. Helander, Z. B. Wang, and Z. H. Lu, Appl. Phys. Lett. 93, 083309 (2008).

${ }^{4}$ C. R. Kagan, D. B. Mitzi, and C. D. Dimitrakopoulos, Science 286, 945 (1999).

${ }^{5}$ R. Zhu, C.-Y. Jiang, B. Liu, and S. Ramakrishna, Adv. Mater. 21, 994 (2009).

${ }^{6}$ H. Proehl, M. Toerker, F. Sellam, T. Fritz, K. Leo, C. Simpson, and K. Müllen, Phys. Rev. B 63, 205409 (2001).

${ }^{7}$ S. Özcan, J. Smoliner, T. Dienel, and T. Fritz, Appl. Phys. Lett. 92, 153309 (2008).

${ }^{8}$ B. Balamurugan and B. R. Mehta, Thin Solid Films 396, 90 (2001).

${ }^{9}$ N. Serin, Semicond. Sci. Technol. 20, 398 (2005).

${ }^{10}$ M. Müller, C. Kübel, and K. Müllen, Chem.-Eur. J. 4, 2099 (1998).

${ }^{11}$ S. Entani, T. Kaji, S. Ideda, T. Mori, Y. Kikuzawa, H. Takeuchi, and K. Saiki, J. Phys. Chem. C 113, 6202 (2009).

${ }^{12}$ Y. Wang, Y. Nie, L. K. Pan, Z. Sun, and C. Q. Sun, Appl. Surf. Sci. 257, 3603 (2011).

${ }^{13}$ B. Balamurugan, B. R. Mehta, and S. M. Shivprasad, Appl. Phys. Lett. 82, 115 (2003).

${ }^{14}$ H. Yorita, K. Otomo, H. Hiramatsu, A. Toyama, T. Miura, and H. Takeuchi, J. Am. Chem. Soc. 130, 15266 (2008).

${ }^{15}$ N. Zhong, H. Shima, and H. Akinaga, Appl. Phys. Lett. 96, 042107 (2010).

${ }^{16}$ R. Waser and M. Aono, Nature Mater. 6, 833 (2007).

${ }^{17}$ R. Dong, D. S. Lee, W. F. Xiang, S. J. Oh, D. J. Seong, S. H. Heo, H. J. Choi, M. J. Kwon, S. N. Seo, M. B. Pyun, M. Hasan, and H. Swang, Appl. Phys. Lett. 90, 042107 (2007). 